\title{
Études/Inuit/Studies
}

\section{SONTAG, Natascha, 2007 Carte Inuktitun Inuit Nunanginni \\ Kanatami. The Inuit Language in Inuit Communities in \\ Canada. La langue inuit dans les communautés Inuit au \\ Canada, Fairbanks, University of Alaska Press.}

\section{Frédéric Laugrand}

\section{Volume 33, numéro 1-2, 2009}

Éducation et transmission des savoirs inuit au Canada

Education and transmission of Inuit knowledge in Canada

URI : https://id.erudit.org/iderudit/044976ar

DOI : https://doi.org/10.7202/044976ar

Aller au sommaire du numéro

\section{Éditeur(s)}

Association Inuksiutiit Katimajiit Inc.

Centre interuniversitaire d'études et de recherches autochtones (CIÉRA)

\section{ISSN}

0701-1008 (imprimé)

1708-5268 (numérique)

Découvrir la revue

Citer ce compte rendu

Laugrand, F. (2009). Compte rendu de [SONTAG, Natascha, 2007 Carte Inuktitun Inuit Nunanginni Kanatami. The Inuit Language in Inuit Communities in Canada. La langue inuit dans les communautés Inuit au Canada, Fairbanks, University of Alaska Press.] Études/Inuit/Studies, 33(1-2), 272-273.

https://doi.org/10.7202/044976ar 


\title{
References
}

GRABURN, Nelson

1974-75 Some Problems in the Understanding of Contemporary Inuit Art, Western Canadian Journal of Anthropology, 4(3): 66-72.

\author{
Emily E. Auger \\ Independent Scholar \\ Ontario, Canada \\ augere@canada.com
}

SONTAG, Natascha

2007 Carte Inuktitun Inuit Nunanginni Kanatami. The Inuit Language in Inuit Communities in Canada. La langue inuit dans les communautés Inuit au Canada, Fairbanks, University of Alaska Press.

Cette carte de Natascha Sontag est sans aucun doute beaucoup plus qu'une simple carte géographique. Des informations figurent au recto comme au verso du document, ce qui impliquera - avis aux intéressé(e)s désireux de l'afficher sur un mur -, d'en acheter au moins deux exemplaires! Sontag s'est probablement inspirée de la remarquable carte Inuit Nunait, publiée en 1995 par l'Alaska Native Language Center, mais son produit final est original et bien réussi.

Au recto, Sontag présente une carte détaillée des langues de l’Arctique canadien. L'auteure a d'abord dessiné les quatre grandes régions de cet espace immense en y faisant figurer de nombreux dialectes perceptibles à l'échelle locale ou régionale. Sur le plan visuel, la présence de nombreux toponymes en syllabique, en inuktitut romain et en anglais, mais aussi des noms de langues et d'autres termes géographiques ne rend pas la lecture très commode. Cet inconvénient comporte toutefois un avantage en ce qu'il oblige le lecteur à se concentrer sur le document. Dès lors, l'information paraît très riche, d'autant plus que Sontag y fait figurer des données statistiques sur les populations et les langues, une légende détaillée et même un exemplaire complet du syllabaire.

Trois critiques peuvent cependant être formulées. D'une part, je ne suis pas pleinement convaincu de toutes les divisions qui sont proposées, en ce sens qu'elles donnent une image très statique des langues et des dialectes du monde inuit, alors que les populations et les parlers sont souvent très diversifiés. Certaines communautés comme Iqaluit ou Arviat comportent en fait plusieurs sous-ensembles où les mélanges sont inévitables. Ici, et en dépit du fait que l'auteure indique les communautés plurilinguistiques par des cercles barrés, on a encore parfois l'impression d'avoir affaire à des ensembles perméables. N'étant ni linguiste ni géographe, j’ignore comment ce défi aurait pu être relevé, mais je pense qu'il faut demeurer très prudent face à cette carte, au risque de croire à tort qu'il n'y aurait que des ensembles homogènes. La présence de l'anglais et du français n’apparaît pas davantage. Les 
déplacements de populations, les relocalisations et aujourd'hui la circulation accrue des Inuit laissent pourtant apparaître des configurations complexes. Je regrette que cette réalité ne figure pas sur la carte.

D’autre part, je m’interroge sur le choix de présenter encore une fois une carte selon la projection Mercator dans la mesure où on aurait pu espérer un peu plus d'effort de la part de l'auteure pour se rapprocher des modèles inuit. Il existe, en effet, d'autres cartes où «l'on ne monte plus au Nord», mais où l'on comprend mieux comment cette région accueille en fait de nombreuses rivières qui s'y déversent, leurs sources étant situées plus au sud, en particulier dans les montagnes rocheuses de l'Alberta.

Enfin, la carte comporte une série de petites lacunes et d'erreurs d'ordre secondaire. L'auteure fait apparaître deux termes pour Pelly Bay alors que le terme de Kugaaruk semble avoir été officiellement adopté. Pangniqtuuq devrait être écrit Panniqtuuq, etc. Sur le plan des langues, on cite le dialecte des Tununirmiut mais pas celui des Uqqurmiut et on ne sait rien du cas du Labrador.

Ceci étant dit, la carte offre plusieurs niveaux d'information et est à ce titre très intéressante à examiner lentement et méticuleusement. Il ne fait aucun doute qu'elle constitue un bon outil pour l'enseignement. Le recto de la carte présente des données assez détaillées et riches sur d'autres aspects de la langue inuit. Sontag y présente quelques explications sous la forme de notes pour que le lecteur puisse mieux lire et saisir sa carte. On y trouve également une section sur les dialectes et les sous-dialectes, une partie consacrée à l'écriture syllabique et à son histoire, et plusieurs paragraphes substantiels sur l'identité des Inuit à travers la langue. Pour finir, l'auteure fournit une courte bibliographie et des références de sites web à consulter. Cette face recto de la carte offre un panorama assez exhaustif qui intéressera surtout ceux et celles qui ne connaissent pas grand-chose des sociétés inuit, mais le spécialiste restera un peu sur sa faim, dans la mesure où les informations fournies restent assez classiques.

En définitive, et hormis quelques critiques, cette carte m'apparaît tout à fait adéquate à des fins d'enseignement et j'en recommande la lecture à tous ceux et celles qui souhaitent mieux connaître les réalités linguistiques et culturelles de l'Arctique canadien où la langue et la culture n'ont de cesse de montrer leur dynamisme. Rien ne remplacera cependant un bon voyage et l'immersion dans des familles qui vivent dans ces communautés.

Frédéric Laugrand Département d'anthropologie Pavillon De Koninck Université Laval Québec (Québec), G1V 0A6, Canada Frederic.Laugrand@ant.ulaval.ca 